\title{
Passive Smoking and Infectious Disease: A Serious Hazard for Cardiovascular System
}

\author{
Aurelio Leone \\ Former Director Department of Internal Medicine, City Hospital Massa, Massa, Italy. \\ Email: reliol@libero.it
}

Received July 27 ${ }^{\text {th }}$ 2011; revised September $11^{\text {th }}, 2011$; accepted October $20^{\text {th }}, 2011$.

\begin{abstract}
Exposure to passive smoking is usually associated with heavy changes in both function and structure of the cardiovascular system at different levels: coronary circulation, heart metabolism, myocardial muscle. These changes may be transient but may have characteristics of irreversibility. Major determinant of cardiovascular alterations is hypoxia due to tobacco products of the environment although a large number of alterations affect immune t-cells and antibody response. All infectious diseases which involve cardiovascular system, including some tropical patterns, particularly Chagas disease, are adversely influenced as a consequence of a continuous although irregular exposure to passive smoking, which worsens the degree of cardiac muscle alterations at different levels like myocardium, coronary arteries and both these structures. Therefore, exposure to passive smoking must be avoided for those individuals suffering from infectious diseases of the heart whatever factor can be responsible.
\end{abstract}

Keywords: Passive Smoking, Tropical Disease, Heart

\section{Introduction}

Exposure to passive smoking is a strong hazard for cardiovascular system [1-22] since it is able to cause a large series of disorders either of functional or structural type as well as to impair cardiac function in those patients affected by tropical diseases which can involve heart and blood vessels [23-25].

Apart from by direct interference of passive smoking with the coronary circulation, which is often involved also by protozoan, the development of cardiovascular alterations can also be regularly seen in experimental findings as a consequence of cardiotoxic chemicals of tobacco smoke in humans and animals.

Estimating the morphological features of cellular damage of myocardium induced by passive smoking could give confusion in terminology and definition of lesion since other factors can cause similar alterations. For example, the term necrosis may be used to define morphologically damage due to coronary circulation involvement, whereas the same term does not recognize a circulatory mechanism when it is due to a directly toxic action on myocardial cells. Moreover, peculiar alterations can be superimposed to those linked with specific alterations caused by tropical agents. Therefore, establishing clearly the meaning of each pathologic pattern observed as well as the mechanism that may be responsible is worthy to be done in an attempt to identify the exact features of cardiac alterations.

This report will describe particularly the main changes that irregular but frequent exposure to passive smoking causes on myocardial and coronary artery cells as well as their possible relationship with those changes caused by tropical agents, particularly restrictive cardiomyopathies.

\section{Passive Smoking and Cardiovascular Damage}

Of the thousands of toxic chemicals which are contained into environmental tobacco smoke, some of these, primarily, play their adverse effects on cardiovascular system.

Four main smoking compounds (Table 1) are usually identified as indicators of passive smoking exposure in different epidemiological surveys $[26,27]$. They are nicotine and its metabolites, carbon monoxide, thiocyanate and polycyclic aromatic substances.

These components, when are absorbed into the blood, act like free or bound plasma constituents and have a different half-life that is about 5 hours for carbon monoxide when it is bound to hemoglobin, 16 hours for cotinine, and 6.5 days for thiocyanates or other alicyclic substances. 
Although inhaled after tobacco manufacturing and burning but not in fresh leaves, carbon monoxide plays a basic role in long-term damage from both active smoking and passive smoking. Nicotine intoxication may occur acutely and depends from both fresh tobacco leaf exposure and exposure to active or passive smoking. Thiocyanate toxicity is too hard to establish since different foods, which are rich in these substances, may adulterate and confuse obtained responses.

Many other chemicals, as already said, may influence adversely heart and blood vessels, but their action is limited when compared to that due to carbon monoxide, nicotine and thiocyanate. On the contrary, these other chemicals, such as ammonia, benzene, 2-naphtylamine, nitrosamines, benzoanthracene and benzopyrene [28] contained in a greater amount in passive smoking - sidestream smoke- have prevailingly heavy carcinogenic effects.

Three main phases characterize passive smoking (Table 2): sidestream smoke which is defined as the smoke that drifts from the end of the lit cigarette, mainstream smoke, which is the smoke inhaled directly through the burning cigarette by the smokers, and then exhaled, mainstream smoke which is the smoke breathed out by the smoker from their lungs. Also the composition of mainstream smoke and exhaled mainstream smoke can likely differ since some of the compounds in smoke are retained by the smokers or otherwise altered by the process of combustion.

Table 1. Chemical compounds of passive smoking determining cardiovascular damage.

\begin{tabular}{ll}
\hline \multicolumn{1}{c}{ Chemicals } & \multicolumn{1}{c}{ Site } \\
\hline $\begin{array}{l}\text { 1) Nicotine and its metabolites } \\
\text { (particularly Cotinine) }\end{array}$ & $\begin{array}{l}\text { Fresh tobacco leaf, Sidestream } \\
\text { smoking } \\
\text { Environmental tobacco, } \\
\text { 2) Carbon monoxide }\end{array}$ \\
$\begin{array}{ll}\text { Burned cigarette } \\
\text { 3) Thiocyanate }\end{array}$ & $\begin{array}{l}\text { Fresh tobacco leaf, Passive } \\
\text { smoking }\end{array}$ \\
4) Polycyclic substances & Environmental tobacco \\
\hline
\end{tabular}

Table 2. Main phases of passive smoking.

\begin{tabular}{cl}
\hline Type of smoking & \multicolumn{1}{c}{ Characteristics } \\
\hline $\begin{array}{c}\text { 1) Sidestream } \\
\text { smoke }\end{array}$ & $\begin{array}{c}\text { the smoke that drifts from the end of the lit ciga- } \\
\text { rette (Carbon monoxide, Nicotine, Carcinogens) }\end{array}$ \\
$\begin{array}{c}\text { 2) Mainstream } \\
\text { smoke }\end{array}$ & $\begin{array}{l}\text { Carbon monoxide, Aromatic amines) } \\
\text { the smoke inhaled by smoked cigarette (Nicotine, }\end{array}$ \\
$\begin{array}{c}\text { 3) Exhaled main- } \\
\text { stream smoke }\end{array}$ & $\begin{array}{l}\text { smoking breathed out by smoker (Carbon mon- } \\
\text { oxide, Carcinogens) }\end{array}$ \\
\hline
\end{tabular}

A large series of clinical and experimental studies conducted in both humans and animals after exposure to environmental tobacco smoke permits to define the type of damage that may affect cardiovascular system.

Observations about passive smoking as a factor, which causes cardiovascular damage, have appeared in the last thirty years. Indoor atmosphere and confined spaces often are polluted by tobacco smoke which is inhaled involuntarily by both smokers and non-smokers. The smokers increase, although slightly, the damage caused by their active smoking, whereas the nonsmokers are damaged heavily against their will.

Experimental and clinical studies conducted on humans and animals following acute or chronic exposure to environmental tobacco smoke allow explaining the harmful effects on the heart and blood vessels.

Acute exposure to passive smoking (from 20 minutes to 8 hours) can result in a decrease in platelet sensitivity to prostacyclin, leading to increased occurrence of thrombosis, a strong effect of carboxyhemoglobin on blood oxygen transport, which induce significant myocardial and endothelial damage [28]. Also cardiac performance with diminished exercise tolerance [29] during acute exposure to environmental tobacco smoke was impaired in both healthy individuals and individuals suffering from a pre-existing myocardial infarction, although with different responses. Healthy individuals showed prolonged time to recovery pre-exercise heart rate, whereas survivors of an acute myocardial infarction had prolonged time to recovery pre-exercise heart rate but also a significant reduction of peak exercise power and ventricular arrhythmias. Higher post-exercise concentration of plasmacarbon monoxide and markedly lower expired-carbon monoxide concentration characterized post-myocardial infarction patients.

Endothelium-dependent coronary artery dilation is impaired in passive smoker women [30]. That should prove that the well characterized endothelial dysfunction in active smokers may occur in passive smokers as well.

Another acute effect of passive smoking on heart function is mediated by a complex set of events due to carbon monoxide inhalation. Indoor polluted by carbon monoxide from passive smoking has a level of this gas usually ranging from $3 \mathrm{ppm}$ (part per million air) to $25 \mathrm{ppm}$, and exposure to $10 \mathrm{ppm}$ over 8 hours results in 1.4\% of the blood hemoglobin occupied as carboxyhemoglobin. Carbon monoxide diminishes the transport of blood oxygen, increases heart rate to maintain an adequate oxygen supply, can cause lifethreatening arrhythmias, and worsens the degree of myocardial ischemia [1,22]. Moreover, chronic exposure to environmental tobacco smoke included a large series of studies [31-33] that prove undoubtedly negative effects on cardiovascular system, particularly due to the 
levels of carboxyhemoglobin. The great majority of patients examined in these studies showed important impairment of those symptoms which characterized their disease, particularly chest pain from angina pectoris and pre-existing myocardial infarction. Such impairment well correlated to carboxyhemoglobin levels as well as artery endothelial dysfunction that are recognized to initiate the development of an atherosclerotic plaque.

Finally, passive smoking increases blood viscosity and hematocrit because of the activation of inflammatory mechanisms and relative hypoxia induced by chronic carbon monoxide exposure [34-37]. In addition, some factors of coagulation fibrinolysis cascade, particularly fibrinogen, increase their blood concentration $[36,37]$ activating the via-thrombus formation.

Also other experimental studies conducted on animals exposed to environmental tobacco smoke showed an increased cardiovascular damage.

Prolonged exposure of animals to carbon monoxide has been shown to induce ultrastructural changes in myocardium with mitochondria damage, focal areas of myocardial necrosis at a different stage, interstitial fibrosis, perivascular infiltrates and punctate hemorrhage. Autophagocytosis and pigmented deposits were also described [38].

Four types of cardiovascular damage are a consequence of acute or chronic exposure to passive smoking as Table 3 shows [39]. A functional damage, usually following acute exposures, is transient and recognizes autonomic system involvement as the main pathogenetic factor. Some cardiovascular parameters rather than others are interested negatively by functional damage: heart rate, systolic blood pressure, exercise tolerance, cardiac rhythm with possible development of ventricular arrhythmias. Also the increased levels of catecholamines share to maintain the damage. Functional damage is especially harmful for those subjects suffering from cardiovascular disease as well as for those who undergo chronic exposure, being autonomic system and catecholamines factors, in themselves, able to have spontaneously detrimental effects on heart and blood vessels.

Table 3. Types of cardiovascular damage caused by passive smoking exposure.

$$
\begin{aligned}
& \text { Functional damage } \\
& \text { Clinical damage } \\
& \text { Anatomical damage } \\
& \text { Combined damages }
\end{aligned}
$$

Clinical damage affects particularly those individuals undergone chronic exposure.
They primarily can show symptoms of myocardial ischaemia. Clinical damage is often associated with an anatomical damage that may act as an underlying disease. Also the association with a transiently functional damage, which may appear after acute environmental tobacco smoke exposure of subjects chronically exposed, worsens clinical damage of those individuals with symptoms related to coronary and cerebrovascular disease.

Finally, anatomical damage is the final and unavoidable result of chronic exposure to passive smoking. Its development is usually late but progressive untill to reach the characteristic of irreversibility.

The factor supporting anatomical damage is a progression of atherosclerotic alterations with, initially, endothelial dysfunction, inflammatory processes and hypoxia. The two latter factors, when they are associated, increase the adverse effects on the heart caused by tropical diseases.

\section{Cardiovascular Damage from Tropical Diseases}

Cardiac involvement due to tropical diseases varies for type and extension depending on etiologic agent, its virulence and health status of affected individual and functional or anatomical characteristics of cardiac muscle. Some factors, as will be widely described, may influence the relationship that exists between passive smoking exposure and tropical diseases.

Commonly defined, tropical diseases are a wide spectrum of alterations that are characterized by a geographical distribution and, usually, vectorial transmission [4042]. They develop primarily, but often uniquely, in tropical or subtropical regions around a circled area involving American, Asian, Australian and African continents as well as Pacific and Indian oceans.

In a large majority of observations tropical diseases are caused by protozoa and metazoa. Table 4 lists the main tropical diseases which may be frequently associated with cardiovascular involvement.

The biological relationship between parasitic agents and their host is yet far to be completely clarified although, usually, unicellular protozoa multiply more rapidly than multicellular parasites and so doing cause involvement of a lot of organs of the body.

Cardiac damage depends on several factors partly related to parasitic activity and partly to heart itself. Usually cardiac muscle is affected by focal or diffuse alterations of inflammatory type that cause an acute or less frequent a chronic myocarditis the most severe and diffuse in the south America is that due to Trypanosoma cruzi, better known as Chagas disease [43,44]. However, whatever etiologic factor acts on the myocardium, the type of cardiac response is an inflammatory reaction that 
Table 4. Main tropical diseases with cardiovascular involvement and etiologic agent.

\begin{tabular}{cc}
\hline Disease & Etiologic agent \\
\hline $\begin{array}{c}\text { American Trypanosomiasis } \\
\text { (Chagas' disease) } \\
\text { Limphatic filariasis }\end{array}$ & Trypanosoma cruzi \\
Malaria & Filaria \\
African Trypanosomiasis & Plasmodium \\
Leishmaniasis & Trypanosoma brucei \\
Toxoplasmosis & Leishmania \\
Pneumocystosis & Toxoplasma condii \\
Amebiasis & Pneumocystis carinii \\
\hline
\end{tabular}

is, usually, worsened by those all factors able to determine hypoxia since oxygen availability is the major determinant of a correct heart function.

A further observation needs to explain either why tropical diseases are more frequent in tropical areas than in other or why some of these may be associated with more markedly cardiovascular impairment. Indeed there exists a strong relationship between climate and tropical diseases in the sense that tropical climate is characterized by heavy rains with formation of stable floods where vector insects may reproduce easily and then transport pathogenic agents.

Yet, socioeconomic status of some population in tropical areas may favour cardiovascular localizations since cardiovascular system strongly feel poor nutrition and hygiene.

Finally, biochemical factors able to cause poor oxygen availability and reduced immuno-response may explain sudden cardiovascular impairment in individuals affected by tropical diseases.

\section{Factors Relating Tropical Diseases to Passive Smoke}

Two main factors link passive smoking exposure and tropical diseases associated with cardiac involvement: hypoxia and immuno-inflammatory phenomena.

Hypoxia is one of the major mechanisms of cardiac muscle damage since metabolic steps of myocardium occur in aerobiosis. Therefore poor oxygen availability adversely influence cardiac muscle structure and function worsening myocardial performance particularly when an underlied heart disease exists.

Moreover, oxygen availability is strictly related to the level of oxygen saturation.
All those pathologic events that influence directly or indirectly the mechanisms of oxygen saturation must be estimated since different percentages of oxygen saturation determine different responses to chemical compounds of passive smoking. Therefore, a large series of diseases as well as those subclinical conditions which need an improvement in oxygen saturation can be impaired as a response to passive smoking.

Particularly, carbon monoxide and all those smoke components defined generically as carcinogens influence oxygen saturation and then some specific diseases. On the contrary, nicotine and cotinine which are those smoke metabolites specifically used to determine environmental tobacco smoke exposure [45] of people are mainly related to functional cardiovascular events.

Adverse effects due to main compounds of passive smoking concern respiratory system, blood, immune function with regard to T-cells and antibody response which feel particularly the alterations in oxygen saturation and metabolism [46,47]. Specifically, respiratory system and blood immune response are closely related to oxygen saturation.

Exposure to tobacco smoke is one of the major causes of both developing and triggering an asthma attack. Moreover, further studies are showing an impaired lung function in those exposed people when they are compared to never smokers not exposed to environmental tobacco $[48,49]$. There is evidence that all respiratory changes characterized by impaired oxygen saturation influence adversely heart function throughout.

Three mechanisms act: alterations of respiratory changes into the lungs, increased levels of carboxyhaemoglobin due to carbon monoxide of environmental smoke, and, then, reduced concentration of oxyhemoglobin into the blood as a consequence of the stronger linkage between carbon monoxide and haemoglobin.

Inflammatory mechanisms triggered by frequent although irregular exposure to passive smoking worsen heart function of those individuals who suffer from a tropical disease that involves this organ.

Immuno T-cells and antibody response [47] are the main factors that are depressed in cardiovascular diseases as an effect of passive smoking exposure.

\section{Conclusions}

Exposure to passive smoking should be considered as a hazard for those people affected by tropical diseases particularly when cardiac muscle is involved by the parasitic alterations.

Passive smoking causes hypoxia that worsens cardiac function and also has depressant effects on immunologic system. 


\section{REFERENCES}

[1] A. Leone, "Cardiovascular Damage from Smoking: A Fact or Belief?” International Journal of Cardiology, Vol. 38, No. 2, 1993, pp. 113-117. doi:10.1016/0167-5273(93)90169-H

[2] C. B. Sherman, "Health Effects of Cigarette Smoking," Clinics in Chest Medicine, Vol. 12, No. 4, 1991, pp. 643658.

[3] A. Leone, "The Heart: A Target Organ for Cigarette Smoking,” Journal of Smoking-Related Disorders, Vol. 3, 1992, pp. 197-201.

[4] US Department of Health, Education, and Welfare, "The Health Consequences of Smoking: A Report of the Surgeon General. Cardiovascular Disease,” US Department of Health and Human Services, Public Health Service Office of Smoking and Health, DHHS Publication NO (PHS) 84-50204, Rockville, 1983.

[5] A. Leone, "Cigarette Smoking and Health of the Heart," Journal of the Royal Society of Health, Vol. 115, No. 6, 1995, pp. 354-355. doi:10.1177/146642409511500605

[6] E. C. Hammond and L. Garfinkel, "Coronary Heart Disease, Stroke and Aortic Aneurysm," Archives of Environmental Health, Vol. 19, 1969, pp. 167-182.

[7] P. E. McBride, "The Health Consequences of Smoking. Cardiovascular Diseases," Medical Clinics of North America, Vol. 76, No. 2, 1992, pp. 333-353.

[8] W. S. Aronow, "Effect of Passive Smoking on Angina Pectoris," The New England Journal of Medicine, Vol. 299, 1978, pp. 21-24. doi:10.1056/NEJM197807062990105

[9] D. Sparrow, T. R. Dawber and T. Colton, “The Influence of Cigarette Smoking on Prognosis after a First Myocardial Infarction,” Journal of Chronic Diseases, Vol. 31, No. 6-7, 1978, pp. 425-432. doi:10.1016/0021-9681(78)90006-1

[10] A. Leone, F. Bertanelli, L. Mori, P. Fabiano and G. Bertoncini, "Ventricular Arrhythmias by Passive Smoking in Patients with Pre-Existing Myocardial Infarction," Journal of the American College Cardiology, Vol. 3, 1992, p. 256(A).

[11] C. Wilhelmsson, J. A. Vedin, D. Elmfeldt, G. Tibblin and L. Wilhelmsen, "Smoking and Myocardial Infarction," Lancet, Vol. 1, No. 7904, 1975, pp. 415-420. doi:10.1016/S0140-6736(75)91488-9

[12] O. Auerbach, H. W Carter, L. Garfinkel and E. C. Hammond, "Cigarette Smoking and Coronary Heart Disease. A Macroscopic and Microscopic Study,” Chest, Vol. 70, No. 6, 1976, pp. 697-705. doi:10.1378/chest.70.6.697

[13] D. D. Reid, P. J. S. Hamilton, P. McCartney and G. Rose, "Smoking and Other Risk Factors in Coronary Heart Disease in British Civil Servants," Lancet, Vol. 308, No. 7993, 1976, pp. 979-984. doi:10.1016/S0140-6736(76)90830-8

[14] S. A. Glantz and W. W. Parmley, "Passive Smoking and Heart Disease," The Journal of the American Medical Association, Vol. 273, No. 13, 1995, pp. 1047-1053. doi:10.1001/jama.273.13.1047

[15] A. J. Wells, "Passive Smoking as a Cause of Heart Disease," Journal of American College Cardiology, Vol. 24, No. 2, 1994, pp. 546-554. doi:10.1016/0735-1097(94)90315-8

[16] C. L. Meinert, S. Forman, D. R. Jacobs and J. Stamler, "Cigarette Smoking as a Risk Factor in Men with a Prior History of Myocardial Infarction," Journal of Chronic Diseases, Vol. 32, 1979, pp. 415-425. doi:10.1016/0021-9681(79)90102-4

[17] S. A. Glantz and W. W. Parmley, "Passive Smoking and Heart Disease: Epidemiology, Physiology, and Biochemistry,” Circulation, Vol. 83, 1991, pp. 1-12.

[18] L. Baer and I. Radichevich, "Cigarette Smoking in Hypertensive Patients. Blood Pressure and Endocrine Responses," American Journal of Medicine, Vol. 78, No. 4, 1985, pp. 564-568. doi:10.1016/0002-9343(85)90396-1

[19] D. S. Celermajer, M. R. Adams, P. Clarkson, J. Robinson, R. McRedie, A. Donald, et al., "Passive Smoking and Impaired Endothelium-Dependent Arterial Dilatation in Healthy Young Adults," The New England Journal of Medicine, Vol. 334, No. 3, 1996, pp. 150-154. doi:10.1056/NEJM199601183340303

[20] A. Leone and M. Lopez, “Oral Contraception, Ovarian Disorders and Tabacco in Myocardial Infarction of Woman,” Pathologica, Vol. 78, 1986, pp. 237-242

[21] S. Pojola, P. Siltanen and M. Romo, "Five-Year Survival of 728 Patients after Myocardial Infarction,” British $\mathrm{He}$ art Journal, Vol. 43, No. 2, 1980, pp. 176-1183. doi:10.1136/hrt.43.2.176

[22] A. Leone, "Relationship between Cigarette Smoking and Other Coronary Risk Factors in Atherosclerosis: Risk of Cardiovascular Disease and Preventive Measures," Current Pharmaceutical Design, Vol. 9, No. 29, 2003, pp. 2417-2423. doi:10.2174/1381612033453802

[23] C. J. Chew, G. M. Ziady, M. J. Raphael, M. Nellen and C. M. Oakley, "Primary Restrictive Cardiomyopathy. NonTropical Endomyocardial Fibrosis and Hypereosi-nophilic Heart Disease,” British Heart Journal, Vol. 39, No. 4, 1977, pp. 399-413. doi:10.1136/hrt.39.4.399

[24] W. A. Thomas, R. W. Randall, E. F. Bland and B. Castleman, "Endocardial Fibroelastosis; a Factor in Heart Disease of Obscure Etiology: A Study of 20 Autopsied Cases in Children and Adults," The New England Journal of Medicine, Vol. 251, 1954, pp. 327-338. doi:10.1056/NEJM195408262510902

[25] J. C. Byrd, "Environmental Tobacco Smoke: Medical and Legal Issues,” Medical Clinics of North America, Vol. 76, No. 2, 1992, pp. 377-397.

[26] R. A. Greenberg, N. J. Haley, R. A. Ersel and F. A. Loda, "Measuring the Exposure of Infants to Tobacco Smoke," The New England Journal of Medicine,, Vol. 310, No. 17, 1984, pp. 1075-1078. doi:10.1056/NEJM198404263101703

[27] D. P. Strachan, M. J. Jarvis and C. Feyerabend, "Passive Smoking, Salivary Cotinine Concentrations and MiddleEar Effusion in 7-Year Old Children,” British Heart Jour- 
nal, Vol. 289, No. 6687, 1989, pp. 1549-1552.

[28] A. Leone, D. Giannini, C. Bellotto and A. Balbarini, "Passive Smoking and Coronary Heart Disease," Current Vascular Pharmacology, Vol. 2, 2004, pp. 175-182. doi:10.2174/1570161043476366

[29] A. Leone, L. Mori, F. Bertanelli, P. Fabiano and M. Filippelli, "Indoor Passive Smoking: Its Effects on Cardiac Performance," International Journal of Cardiology, Vol. 33, No. 2, 1991, pp. 247-252. doi:10.1016/0167-5273(91)90354-R

[30] H. Sumida, H. Watanabe, K. Kugiyama, M. Ohgushi, T. Matsumura and H. Yasue, "Does Passive Smoking Impair Endothelium-Dependent Coronary Artery Dilation in Women?” Journal of the American College Cardiology, Vol. 31, No. 4, 1998, pp. 811-815. doi:10.1016/S0735-1097(98)00010-2

[31] D. P. Strachan, "Predictors of Death from Aortic Aneurysm among Middle-Aged Men: The Whitehall Study," British Journal of Surgery, Vol. 78, No. 4, 1991, pp. 401404. doi:10.1002/bjs.1800780407

[32] W. S. Aronow and S. N. Rokaw, "Carboxyhemoglobin Caused by Smoking Nonnicotine Cigarettes. Effects in Angina Pectoris?” Circulation, Vol. 44, 1971, pp. 782-788.

[33] W. S. Aronow, "Aggravation of Angina Pectoris by Two Percent Carboxyhemoglobin,” American Heart Journal, Vol. 101, No. 2, 1981, pp. 154-157. doi:10.1016/0002-8703(81)90658-X

[34] N. L. Benowitz, "Nicotine and Coronary Heart Disease," Trends in Cardiovasccular Medicine, Vol. 1, No. 8, 1991, pp. 315-321. doi:10.1016/1050-1738(91)90068-P

[35] S. Miyaura, H. Eguchi and J. M. Johnston, "Effect of a Cigarette Smoke Extract on the Metabolism of the Proinflammatoryautacoid, Platelet-Activating Factor," Circulation Research, Vol. 70, No. 2, 1992, pp. 341-347.

[36] T. W. Meade, J. Imeson and Y. Stirling, "Effects of Change in Smoking and Other Characteristics on Clotting Factors and the Risk of Ischemic Heart Disease," Lancet, Vol. 2, No. 8566, 1987, pp. 986-988. doi:10.1016/S0140-6736(87)92556-6

[37] M. C. Stone and J. M. Thorpe, "Plasma-Fibrinogen-A Major Coronary Risk Factor," The Journal of the Royal College of General Practctitioners, Vol. 35, 1985, pp. 565-569.
[38] A. Leone, L. Landini Jr., O. Biadi and A. Balbarini, “Smoking and Cardiovascular System: Cellular Features of the Damage,”s Current Pharmaceutical Design, Vol. 14, No. 18, 2008, pp. 1771-1777. doi:10.2174/138161208784746699

[39] A. Leone, F. Bertanelli, L. Mori, P. Fabiano and A. Battaglia, "Features of Ischaemic Cardiac Pathology Resulting from Cigarette Smoking,” Journal of Smoking-Related Disorders, Vol. 5, 1994, pp. 109-114.

[40] A. W. Melville, “A Problem in Tropical Cardiology,” British Medicine Journal, Vol. 2, No. 5162, 1959, pp. 12731277.

[41] A. A. Zilton and A. C. Guimaraes, "Endomyocardial Fibrosis in Bahia, Brazil,” British Medicine Journal, Vol. 26, No. 6, 1964, pp. 813-820.

[42] J. D. Ball, A. W. Williams and J. N. Davies, "Endomyocardial Fibrosis,” Lancet, Vol. 266, No. 6821, 1954, pp. 1049-1054. doi:10.1016/S0140-6736(54)91619-0

[43] J. M. Hagar and S. H. Rahimtoola, "Chagas' Heart Disease,” Current Problems in Cardiology, Vol. 20, 1995, pp. 825-924.

[44] A. Rassi Jr, A. Rassi and W. C. Little, “Chagas’ Heart Disease,” Clinical Cardiology, Vol. 23, 2000, pp. 883-889.

[45] N. L. Benowitz, "Cotinine as a Biomarker of Environmental Tobacco Smoke Exposure,” Epidemiologic Reviews, Vol. 18, No. 2, 1996, pp. 188-204.

[46] D. P. Strachan and D. G. Cook, "Health Effects of Passive Smoking. Parental Smoking and Childhood Asthma Longitudinal and Case-Control Studies,” Thorax, Vol. 53, No. 3, 1998, pp. 204-212. doi:10.1136/thx.53.3.204

[47] P. G. Holt, "Immune and Inflammatory Function in Cigarettes Smokers,” Thorax, Vol. 42, No. 4, 1987, pp. 241- 249. doi:10.1136/thx.42.4.241

[48] M. A. Masi, J. A. Hanley, P. Ernst and M. R. Becklake, "Environmental Exposure to Tobacco Smoke and Lung Function in Young Adults," American Reviews of Respiratory Disease, Vol. 138, No. 2, 1998, pp. 296-299.

[49] K. H. Svendsen, L. H. Kuller, M. J. Martin and J. K. Ockene, "Effectsof Passive Smoking in the Multiple Risk Factor Intervention Trial,” American Journal of Epidemiology, Vol. 126, 1987, pp. 783-795. 